\title{
Use of rituximab and risk of re-hospitalization for children with neuromyelitis optica spectrum disorder
}

\author{
Sabrina Gmuca ${ }^{1 *}$ DD, Rui Xiao ${ }^{2}$, Pamela F. Weiss ${ }^{1}$, Amy T. Waldman ${ }^{3}$ and Jeffrey S. Gerber ${ }^{2,4}$
}

\begin{abstract}
Background: Treatment algorithms for neuromyelitis optica spectrum disorder (NMOSD) vary, and sparse data exist regarding the impact of initial treatments on disease course. We aimed to determine whether administration of rituximab during first hospitalization reduces 1-year readmission rates.

Methods: We conducted a retrospective cohort study of subjects with NMOSD using the Pediatric Health Information System database from 2005 to 2015. Subjects were ages 1 to 21 years who received glucocorticoids and an ICD-9-CM code indicating neuromyelitis optica (NMO) during first hospitalization. All subjects had at least 12 months of continuous enrollment. The primary exposure was $\geq 1$ rituximab dose during first hospitalization. We tested for the association of rituximab use with all-cause re-hospitalization, the primary outcome, using survival analysis. Re-hospitalization was considered if a hospital admission occurred $>30$ days after initial discharge with exclusion of admissions with re-dosing of rituximab and data were censored at 12 months. Secondary outcomes included time to and median duration of rehospitalization using 25th percentiles of survival time and the Wilcoxon-rank sum test, respectively.
\end{abstract}

Results: Of 180 subjects who met inclusion criteria, $71.7 \%$ were female and the median age was 13 years (IQR: 10, 15). Fifty-two subjects (28.9\%) received rituximab during first hospitalization, and there was an increasing trend in rituximab use over time $(p<0.01)$. Overall, $36.7 \%$ of children were readmitted and time to readmission was a median of 365 days (IQR: 138, 365). Rituximab exposure was not associated with re-hospitalization (adjusted HR: 0.71: 95\% Cl: 0.38, 1.34) nor a reduced time to re-hospitalization. Median duration of re-hospitalization was 2 days shorter in the rituximab exposed group $(p=0.02)$. Receipt of physical therapy, a surrogate marker for neurologic impairment, during first hospitalization was associated with re-admission within 12 months (adjusted HR: 4.81; 95\% Cl: 1.14, 20.29).

Conclusions: Among children with NMOSD, first-line administration of rituximab was not associated with risk of or time to re-hospitalization. Rituximab use was found to be associated with a shorter duration of re-hospitalization. Need for physical therapy during first hospitalization was independently associated with an increased risk of re-admission.

Keywords: Autoimmune, Demyelinating, Neuromyelitis optica, Rituximab

\footnotetext{
*Correspondence: gmucas@email.chop.edu

'Division of Rheumatology, Center for Pediatric Clinical Effectiveness,

Children's Hospital of Philadelphia, Philadelphia, PA, USA

Full list of author information is available at the end of the article
}

(c) The Author(s). 2018 Open Access This article is distributed under the terms of the Creative Commons Attribution 4.0 International License (http://creativecommons.org/licenses/by/4.0/), which permits unrestricted use, distribution, and reproduction in any medium, provided you give appropriate credit to the original author(s) and the source, provide a link to the Creative Commons license, and indicate if changes were made. The Creative Commons Public Domain Dedication waiver (http://creativecommons.org/publicdomain/zero/1.0/) applies to the data made available in this article, unless otherwise stated. 


\section{Background}

Neuromyelitis optica spectrum disorder (NMOSD) is a rare, immune-mediated inflammatory disorder of the central nervous system. This chronic astrocytopathy preferentially targets the optic nerves and spinal cord, resulting in severe and potentially devastating sequelae such as paralysis and blindness. Despite the life-threatening consequences of uncontrolled disease activity, there are no validated treatment strategies for NMOSD [1-3].

NMOSD is rare among children and adolescents, with pediatric onset NMOSD accounting for approximately $3-5 \%$ of all cases [4]. However, early recognition and initiation of treatment is critical for preventing disease relapses in children with NMOSD. Therapeutic management of pediatric NMOSD is largely based on Class IV evidence, extrapolated from studies of adults [5-10]. Immunotherapeutic agents targeting $\mathrm{T}$ and $\mathrm{B}$ cell functions and reducing pro-inflammatory molecules from the peripheral blood circulation have been shown to attenuate disease progression in NMOSD [11]. Rituximab is a chimeric, murine/human monoclonal antibody directed against the CD20 antigen that results in depletion of B cells. Although small, open label [12] and retrospective studies [5] have reported benefits of rituximab in NMOSD, the effectiveness and safety of rituximab for NMOSD remains unclear $[3,13]$. We sought to determine the impact of rituximab on all-cause re-hospitalization for children and young adults with NMOSD. We hypothesized that subjects who received rituximab during their first hospitalization were less likely to be re-admitted within 12 months.

\section{Methods}

We conducted a retrospective cohort study of subjects with NMOSD in the Pediatric Health Information System (PHIS) from January 2005 until December 2015. PHIS is an administrative database of 46 children's hospitals across the United States and represents $85 \%$ of all pediatric hospitalizations at freestanding tertiary care pediatric facilities. All subjects with at least 12 months of continuous enrollment and $\leq 21$ years of age [14] at the time of initial documentation of an ICD-9-CM code for NMO (341.0) were included. The index date was the date of the first admission with an ICD-9-CM code for NMO. We excluded patients less than 1 year of age at the time of diagnosis (because NMOSD is unlikely to present within the first year of life) [15]; or who had an ICD-9-CM code for multiple sclerosis (MS), as this is a distinct disease from NMOSD. We also excluded subjects who did not receive glucocorticoids, which are frequently administered intravenously for optic neuritis or spinal cord deficits in NMOSD. Subjects with an ICD-9CM code for NMOSD but without glucocorticoid exposure likely did not have active neurologic disease and were less likely to be incident cases. The primary exposure was defined as billing for $\geq 1$ dose of rituximab during the initial hospitalization. The primary outcome was all-cause re-hospitalization (binary), a surrogate marker for disease and treatment related morbidity. Re-hospitalization was considered if a hospital admission occurred $>30$ days after initial discharge with exclusion of re-hospitalizations with re-dosing of rituximab and data were censored at 12 months. Secondary outcomes included time to first re-hospitalization and duration of re-hospitalization.

Demographics were summarized by standard descriptive statistics including frequencies and percentages for categorical variables (e.g. sex, race) and by range, median, and interquartile range (IQR) for continuous or count variables. Characteristics between the rituximab exposed and unexposed groups were compared by Wilcoxon rank sum test for medians, and chi-squared test or Fisher's exact test for proportions as appropriate. Time to hospitalization was calculated in days from the initial hospitalization until the first readmission and survival analysis was used to calculate 25th percentiles of time to re-hospitalization based on rituximab exposure. Kaplan-Meier survival curves of re-hospitalization were generated for the rituximab exposed and unexposed groups and compared using the log-rank test. The proportional hazards assumption for the primary exposure was met (Shoenfeld residuals $p=0.58$ ). The univariate Cox proportional hazard regression model was used to evaluate the marginal effect of each covariate on the outcome, including demographics, glucocorticoid type, level of care, physical therapy, plasma exchange (PLEX) and duration of initial hospital stay. Covariates with a $p$-value less than 0.2 were considered in forward selection using the likelihood ratio test to build a multivariable cox regression model with certain variables of interest forced into the model. The proportional hazards assumption was met using Schoenfeld residuals with global test $p=0.31$. According to Cox-Snell residuals, the final model fit the data well. We did not assess competing risks because the data source did not include mortality data. The trend in the proportion of children with NMOSD who received first-line rituximab therapy per calendar year was tested by 'nptrend' command implemented in Stata. All tests considered were two-sided and a significance level of 0.05 was applied. Data analyses were performed using STATA 14 (STATA Corp, College Station TX).

\section{Results}

Of the 351 eligible subjects with at least one code for NMO, 180 subjects met study inclusion and exclusion criteria (Fig. 1). Table 1 shows patient characteristics. The majority of subjects were female (71.7\%) and a little 


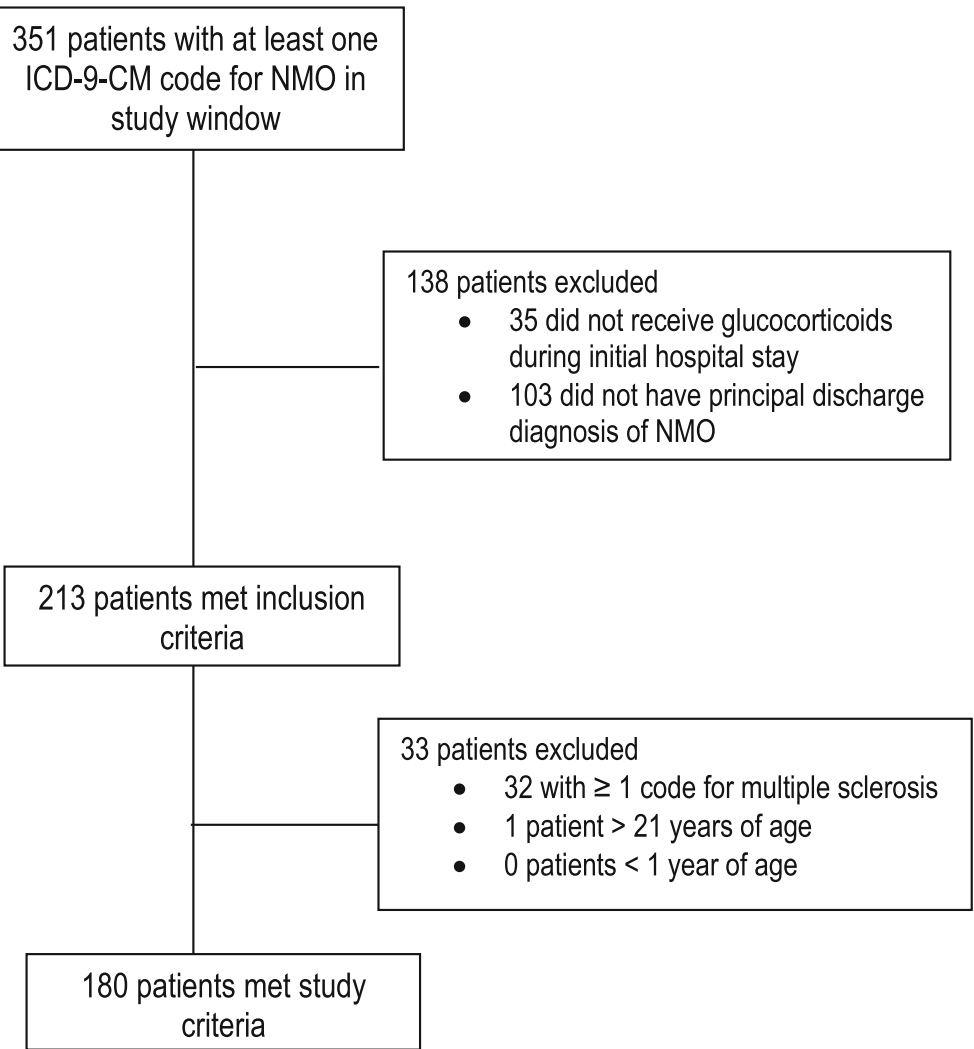

Fig. 1 Flow diagram outlining inclusion/exclusion criteria

less than half were Caucasian (40.0\%). Median age at time of first hospitalization was 13 years (IQR: 10.0, 15. $0)$ and median duration of initial hospitalization was 6 days (IQR: $3.0,12.0$ ). Only 3 subjects had a concomitant systemic rheumatologic condition. A total of 16 subjects $(8.9 \%)$ received oral glucocorticoids only; 76 (42.2\%) received methylprednisolone only; and 88 (48. 9\%) received a combination of oral and intravenous glucocorticoids. Table 2 lists the proportion of subjects who received each of the glucocorticoid sparing therapies administered during initial hospitalization. The most common were plasma exchange (PLEX) (31.1\%), rituximab (28.9\%), and intravenous immunoglobulin (IVIg) (18.3\%); 61 subjects (33.9\%) did not receive any glucocorticoid sparing therapies. Of those subjects who received rituximab $(n=52), 20$ subjects $(38.5 \%)$ also received plasma exchange and 23 subjects (44.2\%) received rituximab without any other non-glucocorticoid immunosuppressive therapy.

Fifty-two subjects (28.9\%) received rituximab during their first hospitalization with an increasing trend over time $(p<0.01)$ (Fig. 2). Comparing subjects in the rituximab exposed to unexposed, the proportion of non-Caucasian subjects in the rituximab group was greater than in the unexposed group $(70.2 \%$ vs. 42. $0 \%$ ). Overall, $36.7 \%$ of subjects were re-hospitalized at least once within the first year after diagnosis (Table 1). Kaplan-Meier estimates comparing the probability of rehospitalization for children with NMOSD treated with rituximab versus those treated with alternative therapies showed no statistically significant difference between groups (log-rank test $p=0.76$ ) (Fig. 3). Median duration of re-hospitalization was 2 days (IQR: 1.0, 4.0) in subjects administered first-line rituximab compared to 4 days (IQR: $2.0,6.0)$ in those subjects who did not receive rituximab $(p=0.02) .25 \%$ of rituximab exposed subjects were re-hospitalized within 137 days (95\% CI: 65, 295) and 25\% of rituximab unexposed were re-hospitalized within 134 days (95\% CI: 92, 192), indicating a similar time to rehospitalization irrespective of rituximab exposure.

In univariate Cox regression modeling, the relative hazard of all-cause re-hospitalization in the first 12 months was 1.08 (95\% CI: $0.64,1.83)$ for those treated with rituximab compared to those not treated with rituximab $(p=0.77)$. In the final multivariate model (Table 3 ), the hazard ratio (HR) of re-hospitalization within 12 months was 0.71 (95\% CI: 0.38, 1.34) for those children who received rituximab compared to those 
Table 1 Patient characteristics

\begin{tabular}{|c|c|c|c|}
\hline & Total $(n=180)$ & $\begin{array}{l}\text { Rituximab exposed } \\
(n=52)\end{array}$ & $\begin{array}{l}\text { Rituximab unexposed } \\
(n=128)\end{array}$ \\
\hline \multicolumn{4}{|l|}{ Demographics } \\
\hline Female, $n(\%)$ & $129(71.7)$ & $43(82.7)$ & $86(67.2)$ \\
\hline Age, median (IQR) & $13.0(10.0,15.0)$ & $13.0(10.0,16.0)$ & $12.5(9.0,15.0)$ \\
\hline Caucasian $^{\mathrm{a}}, n(\%)$ & $72(48.9)$ & $14(29.8)$ & $58(58.0)$ \\
\hline \multicolumn{4}{|l|}{ Region, $n(\%)$} \\
\hline Northeast & $29(16.1)$ & $9(17.3)$ & $20(15.6)$ \\
\hline Midwest & $29(16.1)$ & $8(15.4)$ & $21(16.4)$ \\
\hline South & $98(54.4)$ & $30(57.7)$ & $68(53.1)$ \\
\hline West & $24(13.3)$ & $5(9.6)$ & $19(14.8)$ \\
\hline Systemic lupus erythematosus, $n(\%)$ & $2(1.1)$ & $2(3.9)$ & $0(0.0)$ \\
\hline Sjögren syndrome, $n$ (\%) & $1(0.6)$ & $1(1.9)$ & $0(0.0)$ \\
\hline ICU status, n (\%) & $31(17.2)$ & $11(21.2)$ & $20(15.6)$ \\
\hline Length of stay (days), median (IQR) & $6(3.0,12.0)$ & $7.0(3.0,17.0)$ & $6.0(3.0,11.0)$ \\
\hline \multicolumn{4}{|l|}{ Primary outcome } \\
\hline Re-hospitalization in 12 months, $n$ (\%) & $66(36.7)$ & $20(38.5)$ & $46(35.9)$ \\
\hline \multicolumn{4}{|l|}{ Secondary outcomes } \\
\hline Length of re-hospitalization (days), median (IQR) & $3.0(1.0,5.0)$ & $2.0(1.0,4.0)$ & $4.0(2.0,6.0)$ \\
\hline Time to re-hospitalization (days), median (IQR) & $365(138,365)$ & $365(138,365)$ & $365(137,365)$ \\
\hline
\end{tabular}

ICU intensive care unit, IQR interquartile range

${ }^{a}$ Race was missing for 33 subjects. Rituximab exposure was defined as having received at least one dose of rituximab during initial hospitalization with a documented ICD-9-CM code for neuromyelitis optica (341.0)

who did not $(p=0.29)$. Receipt of physical therapy was independently associated with an increased risk of rehospitalization within the first 12 months (HR: 4.82; 95\% CI: $1.14,20.29)$.

\section{Discussion}

Using a database of 46 freestanding children's hospitals, we assessed the impact of first-line rituximab use on children and adolescents with NMOSD. We did not observe an association between rituximab use and risk of

Table 2 Proportion of subjects administered non-glucocorticoid treatment regimens during first hospitalization $(n=180)$

\begin{tabular}{ll}
\hline Treatment & $n,(\%)$ \\
\hline Plasma exchange (PLEX) $^{\mathrm{a}}$ & $56(31.1)$ \\
Rituximab $^{b}$ & $52(28.9)$ \\
Intravenous immunoglobulin (IVlg) $^{c}$ & $33(18.3)$ \\
Mycophenolate mofetil & $23(12.8)$ \\
Azathioprine & $15(8.3)$ \\
Cyclophosphamide & $10(5.6$ \\
Methotrexate & $1(0.6)$
\end{tabular}

Legend. Subjects were able to contribute to more than one treatment regimen therefore total does not equal $100 \%$

apLEX + rituximab $(n=20)$

${ }^{\mathrm{b}}$ Rituximab only, without any of the above listed non-glucocorticoid agent $(n=23)$

${ }^{\mathrm{I}} \mathrm{VIg}+$ rituximab $(n=8)$ all-cause re-hospitalization or time to all-cause rehospitalization. Although first-line rituximab use was associated with a shorter duration of re-hospitalization, the clinical significance of this is unclear. Receipt of physical therapy was independently associated with an increased risk of re-hospitalization.

This study, to our knowledge, comprises the largest cohort of pediatric subjects with NMOSD administered rituximab first-line. Previous work has largely focused on the use of rituximab as maintenance therapy in the treatment of NMOSD, with a limited number of studies examining the efficacy of first-line rituximab use $[1,5$, 16]. Zephir et al. reported a retrospective analysis of 32 adults with NMOSD who received first-line rituximab and found that at the end of the follow-up period (mean 28.7 months), $84.3 \%$ were relapse free [1]. Olivieri et al. described the case of a 9-year-old girl who received rituximab along with administration of methylprednisolone and plasma exchange and had a slow but full neurologic recovery [16]. Longoni et al. performed a retrospective cohort study of 5 pediatric subjects (all aquaporin-4 antibody positive) with NMOSD treated with first-line rituximab; $60 \%(3 / 5)$ had a relapse of their disease within 12 months of initial rituximab therapy but relapses were reportedly less severe than prior to treatment and all patients demonstrated neurologic improvements by the end of the followup period [5]. 


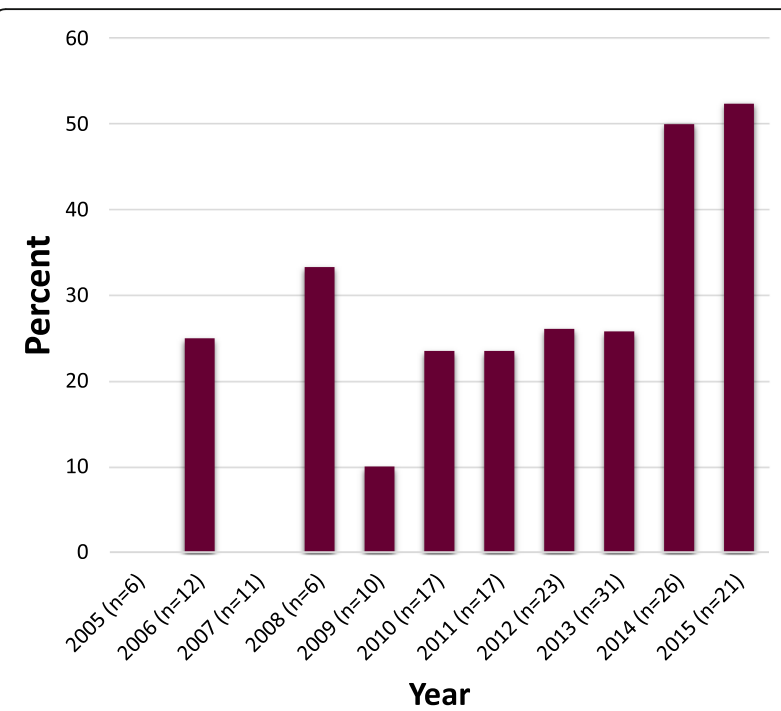

Fig. 2 Proportion of subjects with NMOSD administered first-line rituximab per year $(n=180)$

Our findings did not show a statistically significant reduction in risk of all-cause re-hospitalization with firstline rituximab use. However, it is possible that our study was underpowered or did not fully account for residual confounding by indication, resulting in negative findings. We did find that the duration of re-hospitalization, however, was statistically significantly shorter among those subjects who received rituximab therapy upfront. This suggests, similar to the findings of Longoni et al., that even for rituximab exposed children who experienced disease flares within 12 months, the severity of their flares was less. Therefore, in light of the potential for significant morbidity and mortality in children with newly diagnosed NMOSD, first-line rituximab should be carefully considered.

Receipt of physical therapy during initial hospitalization was found to be an independent predictor of rehospitalization within 12 months. Likely, those children necessitating physical therapy during their initial hospital encounter, had a more severe presentation of their NMOSD. Children with physical therapy services likely incurred significant morbidity such as paralysis and for this reason alone would be more likely to have subsequent complications leading to re-admissions. Unfortunately, such insults cannot be reversed even with first-line rituximab use.

With respect to demographics, we found that nonCaucasian subjects were more likely to receive rituximab during their first hospitalization. Previous research has shown that certain races, specifically Afro-Caribbeans, have greater relapse rates [17]. This may drive providers to treat non-Caucasian patients more aggressively and favor first-line rituximab in this patient population.

This study has limitations. First, there is a possibility of misclassification bias because the NMO ICD-9-CM code has not been validated. However, given the specificity of language for this code and the rarity of this disease, we believe it would be unlikely for practitioners to incorrectly assign patients an ICD-9-CM code for NMO. We also attempted to increase the specificity of the code by requiring receipt of glucocorticoids and excluding subjects with codes for MS. We may, however, have failed to capture all patients with NMOSD.

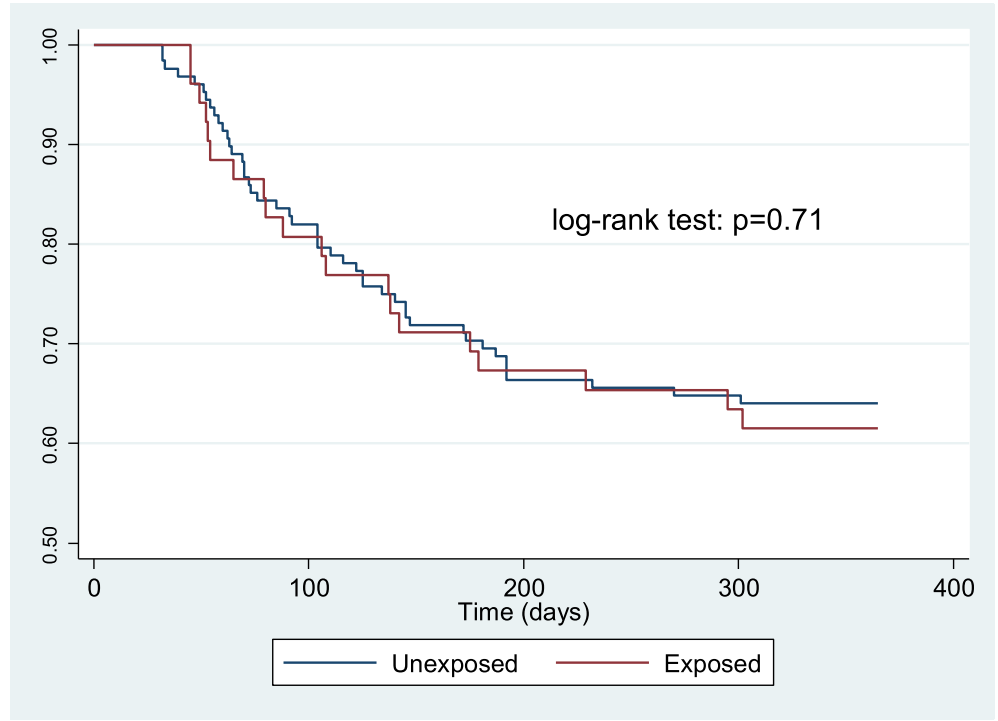

Fig. 3 Kaplan-Meier estimates of re-hospitalization by rituximab exposure 
Table 3 Multivariate Cox proportional hazards for rehospitalization within 12 months

\begin{tabular}{llll}
\hline Covariate & Adjusted HR & $95 \% \mathrm{Cl}$ & P-value \\
\hline Rituximab exposure & 0.71 & $0.38,1.34$ & 0.29 \\
Male gender & 0.68 & $0.35,1.32$ & 1.32 \\
Caucasian & 0.90 & $0.50,1.61$ & 0.72 \\
Region (reference: Midwest) & & & \\
$\quad$ Northeast & 0.74 & $0.28,1.91$ & 0.53 \\
$\quad$ South & 0.70 & $0.33,1.47$ & 0.35 \\
$\quad$ West & 1.09 & $0.40,2.95$ & 0.87 \\
Length of Initial Stay (days) & 0.98 & $0.94,1.03$ & 0.41 \\
ICU status & 1.41 & $0.61,3.25$ & 0.43 \\
Receipt of PT & 4.81 & $1.14,20.29$ & 0.03 \\
Receipt of PLEX & 1.01 & $0.49,2.08$ & 0.98 \\
Glucocorticoid (reference: oral only) & & \\
$\quad$ Intravenous only & 0.88 & $0.28,2.72$ & 0.82 \\
$\quad$ Oral + intravenous & 1.72 & $0.57,5.18$ & 0.33
\end{tabular}

Physical therapy (PT) exposure defined as at least one procedure code for PT during initial hospitalization. Oral glucocorticoids defined as at least one billing code for dexamethasone, hydrocortisone, prednisone or prednisolone $\mathrm{Cl}$ confidence interval, ICU intensive care unit, PLEX plasma exchange

Additionally, the relatively recent discovery of the antimyelin oligodendrocyte glycoprotein (MOG) antibodies [18-20] could lead to misclassification bias in our study. During the study interval, testing for MOG would not have been available to providers and would not affect treatment decision making; however, subjects with NMOSD who are seropositive for anti-MOG, have more favorable outcomes and tend to be glucocorticoid responsive [19]. Therefore, dependent on the number of subjects with anti-MOG positivity in this study, this could bias our primary outcome towards the null and, in part, explain our study's negative findings. Second, we likely did not identify all outcomes of interest by using retrospective data; however, by only including patients with 12 months of available follow-up time in the database we aimed to minimize any outcome bias. Third, by using claims data we were limited in our ability to assess clinical severity or aquaporin-4 antibody status in this cohort of patients with NMOSD. Of note, laboratory detection of aquaporin- 4 antibodies is a send-out test for most institutions with a turn-around-time of 5-8 days; thus most providers make treatment decisions for a patient's initial attack prior to receiving the test results. Fourth, there may have been residual confounding by indication. However, we attempted to address confounding by indication by adjusting for receipt of PLEX, receipt of PT, duration of initial hospitalization, and ICU status in our final multivariate logistic regression model. We did not find that these indicators of disease severity were independently associated with re-hospitalization.

\section{Conclusions}

Among children with NMOSD, first-line rituximab therapy was not associated with a decreased risk of or time to all-cause re-hospitalization within 12 months. However, first-line rituximab use was associated with a decreased duration of re-hospitalization, suggesting its possible role in mitigating the severity of subsequent disease relapses. Further research is warranted to determine the long-term effects of first-line rituximab on morbidity and mortality, which will help guide the optimal treatment regimen for NMOSD taking into consideration disease progression, adverse events, and re-admissions.

\section{Abbreviations}

HR: Hazard ratio; ICU: Intensive care unit; IVIg: Intravenous immunoglobulin; MS: Multiple sclerosis; NMO: Neuromyelitis optica; NMOSD: Neuromyelitis optica spectrum disorder; PHIS: Pediatric health information system; PLEX: Plasma exchange; SLE: Systemic lupus erythematosus

\section{Acknowledgements \\ The authors thank Emily R. Schriver for her assistance with data management.}

\section{Funding \\ Dr. Gmuca is supported by the National Institute of Health Rheumatology Research Training Grant T32-AR007442-29. Dr. Waldman has received funding from the National Institute of Neurologic Disorders and Stroke Grant K23- NS069806. This study was funded by the Children's Hospital of Philadelphia Center for Pediatric Clinical Effectiveness (CPCE) Pilot Grant Program.}

\section{Availability of data and materials}

The datasets used and/or analyzed during the current study are available from the corresponding author on reasonable request.

\section{Authors' contributions}

SG, PW, AW, JG are responsible for the conception and design of the work. SG is responsible for data collection, data analysis and drafting of the manuscript. SG, PW, AW, JG contributed to the data interpretation. All the authors are responsible for critical revision of the article and gave final approval of the version to be published.

\section{Ethics approval and consent to participate}

This study was determined by The Children's Hospital of Philadelphia institutional review board to not meet the requirements of human subjects research and therefore consent was not required.

Competing interests

The authors declare that they have no competing interests.

\section{Publisher's Note}

Springer Nature remains neutral with regard to jurisdictional claims in published maps and institutional affiliations.

\section{Author details}

${ }^{1}$ Division of Rheumatology, Center for Pediatric Clinical Effectiveness, Children's Hospital of Philadelphia, Philadelphia, PA, USA. 'Department of Biostatistics, Epidemiology and Informatics, Perelman School of Medicine at the University of Pennsylvania, Philadelphia, PA, USA. ${ }^{3}$ Division of Neurology, Children's Hospital of Philadelphia, Philadelphia, PA, USA. ${ }^{4}$ Division of Infectious Diseases, Center for Pediatric Clinical Effectiveness, Children's Hospital of Philadelphia, Philadelphia, PA, USA. 
Received: 8 January 2018 Accepted: 9 April 2018

/ Published online: 17 April 2018

\section{References}

1. Zephir H, Bernard-Valnet R, Lebrun C, Outteryck O, Audoin B, Bourre B, Pittion S, Wiertlewski S, Ouallet JC, Neau JP, et al. Rituximab as first-line therapy in neuromyelitis optica: efficiency and tolerability. J Neurol. 2015; 262:2329-35.

2. Kimbrough DJ, Fujihara K, Jacob A, Lana-Peixoto MA, Leite MI, Levy M, Marignier R, Nakashima I, Palace J, de Seze J, et al. Treatment of neuromyelitis optica: review and recommendations. Mult Scler Relat Disord. 2012;:180-7.

3. Kim SH, Kim HJ. Rituximab in Neuromyelitis Optica Spectrum disorders: why not as first-line therapy. JAMA Neurol. 2017;74:482.

4. Tenembaum S, Chitnis T, Nakashima I, Collongues N, McKeon A, Levy M, Rostasy K. Neuromyelitis optica spectrum disorders in children and adolescents. Neurology. 2016;87:S59-66.

5. Longoni G, Banwell B, Filippi M, Yeh EA. Rituximab as a first-line preventive treatment in pediatric NMOSDs: preliminary results in 5 children. Neurol Neuroimmunol Neuroinflamm. 2014;1:e46.

6. Torres J, Pruitt A, Balcer L, Galetta S, Markowitz C, Dahodwala N. Analysis of the treatment of neuromyelitis optica. J Neurol Sci. 2015;351:31-5.

7. Evangelopoulos ME, Andreadou E, Koutsis G, Koutoulidis V, Anagnostouli M, Katsika P, Evangelopoulos DS, Evdokimidis I, Kilidireas C. Treatment of neuromyelitis optica and neuromyelitis optica spectrum disorders with rituximab using a maintenance treatment regimen and close CD19 B cell monitoring. A six-year follow-up. J Neurol Sci. 2017;372:92-6.

8. Radaelli M, Moiola L, Sangalli F, Esposito F, Barcella V, Ferre L, Rodegher M, Colombo B, Fazio R, Martinelli V, Comi G. Neuromyelitis optica spectrum disorders: long-term safety and efficacy of rituximab in Caucasian patients. Mult Scler. 2016;22:511-9.

9. Kim SH, Huh SY, Lee SJ, Joung A, Kim HJ. A 5-year follow-up of rituximab treatment in patients with neuromyelitis optica spectrum disorder. JAMA Neurol. 2013;70:1110-7.

10. Kim SH, Jeong IH, Hyun JW, Joung A, Jo HJ, Hwang SH, Yun S, Joo J, Kim HJ. Treatment outcomes with rituximab in 100 patients with neuromyelitis optica: influence of FCGR3A polymorphisms on the therapeutic response to rituximab. JAMA Neurol. 2015;72:989-95.

11. Bienia B, Balabanov R. Immunotherapy of neuromyelitis optica. Autoimmune Dis. 2013;2013:741490

12. Cree BA, Lamb S, Morgan K, Chen A, Waubant E, Genain C. An open label study of the effects of rituximab in neuromyelitis optica. Neurology. 2005;64:1270-2

13. Damato V, Evoli A, lorio R. Efficacy and safety of rituximab therapy in neuromyelitis optica spectrum disorders: a systematic review and metaanalysis. JAMA Neurol. 2016;73:1342-8.

14. Hardin AP, Hackell JM. Age limit of pediatrics. Pediatrics. 2017:140:e20172151.

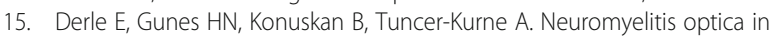
children: a review of the literature. Turk J Pediatr. 2014;56:573-80.

16. Olivieri G, Nociti V, lorio R, Stefanini MC, Losavio FA, Mirabella M, Mariotti P. Rituximab as a first-line treatment in pediatric neuromyelitis optica spectrum disorder. Neurol Sci. 2015;36:2301-2.

17. Tackley G, O'Brien F, Rocha J, Woodhall M, Waters P, Chandratre S, Halfpenny C, Hemingway C, Wassmer E, Wasiewski W, et al. Neuromyelitis optica relapses: race and rate, immunosuppression and impairment. Mult Scler Relat Disord. 2016;7:21-5.

18. Chalmoukou K, Alexopoulos H, Akrivou S, Stathopoulos P, Reindl M, Dalakas MC. Anti-MOG antibodies are frequently associated with steroid-sensitive recurrent optic neuritis. Neurol Neuroimmunol Neuroinflamm. 2015;2:e131.

19. Kitley J, Woodhall M, Waters P, Leite MI, Devenney E, Craig J, Palace J, Vincent A. Myelin-oligodendrocyte glycoprotein antibodies in adults with a neuromyelitis optica phenotype. Neurology. 2012;79:1273-7.

20. Waters P, Woodhall M, O'Connor KC, Reindl M, Lang B, Sato DK, Junynczyk M, Tackley G, Rocha J, Takahashi T, et al. MOG cell-based assay detects non-MS patients with inflammatory neurologic disease. Neurol Neuroimmunol Neuroinflamm. 2015;2:e89.

\section{Ready to submit your research? Choose BMC and benefit from:}

- fast, convenient online submission

- thorough peer review by experienced researchers in your field

- rapid publication on acceptance

- support for research data, including large and complex data types

- gold Open Access which fosters wider collaboration and increased citations

- maximum visibility for your research: over $100 \mathrm{M}$ website views per year

At BMC, research is always in progress.

Learn more biomedcentral.com/submissions 